\title{
Perceptions and attitudes toward clinical trials in adolescent and young adults with cancer: a systematic review
}

This article was published in the following Dove Press journal:

Adolescent Health, Medicine and Therapeutics

\author{
Victoria Forcina' \\ Branavan Vakeesan' \\ Chelsea Paulo' \\ Laura Mitchell' \\ Jennifer AH Bell ${ }^{2}$ \\ Seline Tam' \\ Kate Wang' \\ Abha A Gupta1,3,4 \\ Jeremy Lewin ${ }^{1,3,5}$
}

'Adolescent and Young Adult Program, Princess Margaret Cancer Centre, University of Toronto, Toronto, ON, Canada; ${ }^{2}$ Joint Center for Bioethics, University of Toronto, Toronto,

ON, Canada; ${ }^{3}$ Division of Medical Oncology and Hematology, Princess Margaret Cancer Centre, University of Toronto, Toronto, ON, Canada; ${ }^{4}$ Division of Hematology/Oncology, Hospital for Sick Children, University of Toronto, Toronto, ON, Canada; ${ }^{5}$ ONTrac, Peter MacCallum Cancer Centre, Melbourne, VIC, Australia
Correspondence: Jeremy Lewin ONTrac at Peter Mac Victorian Adolescent \& Young Adult Cancer Service, Peter MacCallum Cancer Centre, 305 Grattan Street, Melbourne, VIC 3000 , Australia

Tel +6I 385596800

Email Jeremy.Lewin@petermac.org
Purpose: Although cancer clinical trials (CT) offer opportunities for novel treatments that may lead to improved outcomes, adolescents and young adults (AYA) are less likely to participate in these trials as compared to younger children and older adults. We aimed to identify the perceptions and attitudes toward CT in AYA that influence trial participation.

Materials and methods: A systematic review of cancer literature was conducted that assessed perceptions and attitudes toward CT enrollment limited to AYA patients (defined as age 15-39). We estimated the frequency of identified themes by pooling identified studies.

Results: In total, six original research articles were identified that specifically addressed perceptions or attitudes that influenced CT participation in AYA patients. Three studies were conducted at pediatric centers - one at an AYA unit, one at an adult cancer hospital, and one was registry based. Major themes identified for CT acceptability included: hope for positive clinical affect, altruism, and having autonomy. Potential deterrents included: prolonged hospitalization, worry of side effects, and discomfort with experimentation.

Conclusion: Limited information is available with regard to the perceptions and attitudes toward CT acceptability among AYA patients, especially those treated at adult cancer centers, which prevents generalization of data and themes. Future research assessing strategies for understanding and supporting CT decision-making processes among AYA represents a key focus for future funding to improve $\mathrm{CT}$ enrollment.

Keywords: adolescent and young adult, clinical trials, barriers, psychosocial, cancer

\section{Introduction}

Adolescents and young adults (AYA) account for approximately 5\% of the adult cancer population, with over 70,000 AYA aged 15-39 years diagnosed annually in the USA, ${ }^{1}$ with the majority being treated at adult cancer centers. For a variety of reasons, AYA are the least represented age cohort in cancer clinical trials (CT) internationally. ${ }^{2,3}$ Improving trial accrual is critical because low recruitment impedes the early introduction of effective treatments into the clinical setting. ${ }^{4}$ The impact of trials on improving outcome is an issue of debate ${ }^{.5}$ however, it does not negate the need to understand systematic recruitment barriers based on age.

Impediments to AYA trial accrual have been well documented and include factors related to health care professionals and institutions as well as individual patients. ${ }^{4}$ Structural barriers such as place of care, ${ }^{2,8}$ eligibility criteria, ${ }^{2}$ lack of age- and stagespecific CT, ${ }^{9-11}$ and access to medical insurance ${ }^{10,12}$ are most often cited as impediments to accrual. Although both cancer patients and the general public are generally 
supportive, in theory, of acting as research subjects in $\mathrm{CT},{ }^{13}$ fewer people are willing to actually participate in randomized CT themselves. ${ }^{14,15}$ What remains understudied are the perceptions held by AYA patients that may influence CT participation. It is likely that life experiences and developmental stage contribute to the decision-making process for cancer trials as they do in other health care situations. A systematic review of barriers to $\mathrm{CT}$ enrollment has been conducted in both elderly ${ }^{16}$ and adults with cancer. ${ }^{17}$ We aimed to conduct a systematic review of studies limited to AYA patients which assessed attitudes and beliefs that influence cancer CT enrollment to prioritize areas for future study and intervention.

\section{Materials and methods Search strategy and selection criteria}

A systematic search of cancer literature was conducted according to the Preferred Reporting Items for Systematic Reviews and Meta-Analyses (PRISMA) guidelines in order to identify information pertaining to AYA decision-making processes for CT enrollment. The authors VF, BV, and CP searched the following databases that pertain to AYA cancer patients and CT participation: Medline (1946 to May 2017); MEDLINE Epub (May 2017); EMBASE (1974 to May 2017); PsychINFO (1806 to May 2017); Cochrane CT library (2005 to May 2017); and Cochrane SR Libraries. The studies considered were limited to those written in English and to those focused on adolescents aged 13-17 years, or adults aged 18-64 years. The databases were searched after consensus of team members and with advice from the institutional information specialist using MeSH terms/key words such as "neoplasms," "adolescent" or teenage" or TYA or AYA," "cancer patient," "attitude," "patient participation," "attitude to health," "decision making," "refusal to participate," "enroll," "clinical trials," "surveys and questionnaires," "registries," and "survey." In total, 2,330 EMBASE, 1,528 Medline, 148 MEDLINE Epub, 217 PsychoINFO, 1,183 Cochrane CT library, and 533 Cochrane SR library articles were identified using this process. BV, CP, and VF supplemented the search by reviewing the bibliographies of the final papers, but yielded no additional studies. The final studies met the following eligibility criteria: 1) original research, 2) inclusive of an AYA population (defined as age range 15-39), ${ }^{18}$ 3 ) content addressing patient-identified barriers to participation in cancer CT, and 4) data-collection methodology using semi-structured interview, focus-group study, patient survey, or questionnaire. Studies were excluded if they 1) did not pertain to cancer $\mathrm{CT}, 2$ ) involved cancer screening or cancer prevention, 3) did not include AYA patient demographic, or 4) were conducted solely among doctors or parents of AYA without corresponding data of the AYA themselves.

\section{Data abstraction and validity assessment}

$\mathrm{BV}$ and $\mathrm{CP}$ independently extracted the final included studies and appraised content using the COREQ checklist. ${ }^{19} \mathrm{~A}$ coding template was developed on the basis of previously published data to categorize key barriers and facilitators to CT enrollment. ${ }^{17}$ Any discrepancy in the finalized list was discussed with JL, who acted as the adjudicator for inclusion. The coding was subjectively divided into the following headings: perceived barriers (protocol-related barriers; patient-related barriers) and perceived facilitators. The dominant themes were those agreed upon by at least three of the authors of this article.

\section{Statistical analysis}

Descriptive statistics were used to report demographic and clinical data and are presented as frequencies.

\section{Results}

A full literature search identified 4,412 unique articles of which, based on abstract screening, 155 studies were found to be relevant according to the inclusion/exclusion criteria. Of these studies, 149 were excluded because they were not original research $(n=10)$, did not include AYA patient demographic $(n=133)$, did not pertain to cancer CTs $(n=3)$, or were of the wrong informant (i.e., parent) $(n=2)$. One study that met all inclusion/exclusion criteria was not included because content focused on suggestions for improving the informed consent process. ${ }^{20} \mathrm{~A}$ total of six studies that investigated perceptions of AYA with regard to CTs were selected for the final analyses. The PRISMA flow diagram is shown in Figure 1. In terms of study design, three of the six studies were conducted using questionnaires/surveys ${ }^{21-23}$ and three using semistructured interviews. ${ }^{24-26}$ Table 1 summarizes study demographics and methodological design of selected studies. Three of the studies were conducted in pediatric institutes, ${ }^{21,24,25}$ two in adult centers, ${ }^{22,23}$ and one in an AYAspecific clinic. ${ }^{26}$ The trial phase was only specified in three of the six studies: two were specific to Phase III trials ${ }^{25,26}$ and one was specific to a Phase I trial. ${ }^{24}$ In addition to exploring the attitudes of AYAs toward CTs, two of the studies investigated the attitudes of parents, ${ }^{21,25}$ health care professionals, ${ }^{26}$ and healthy college students. ${ }^{22}$

Barriers to, and incentives for, AYA trial participation are summarized in Table 2 . The most commonly cited reason 


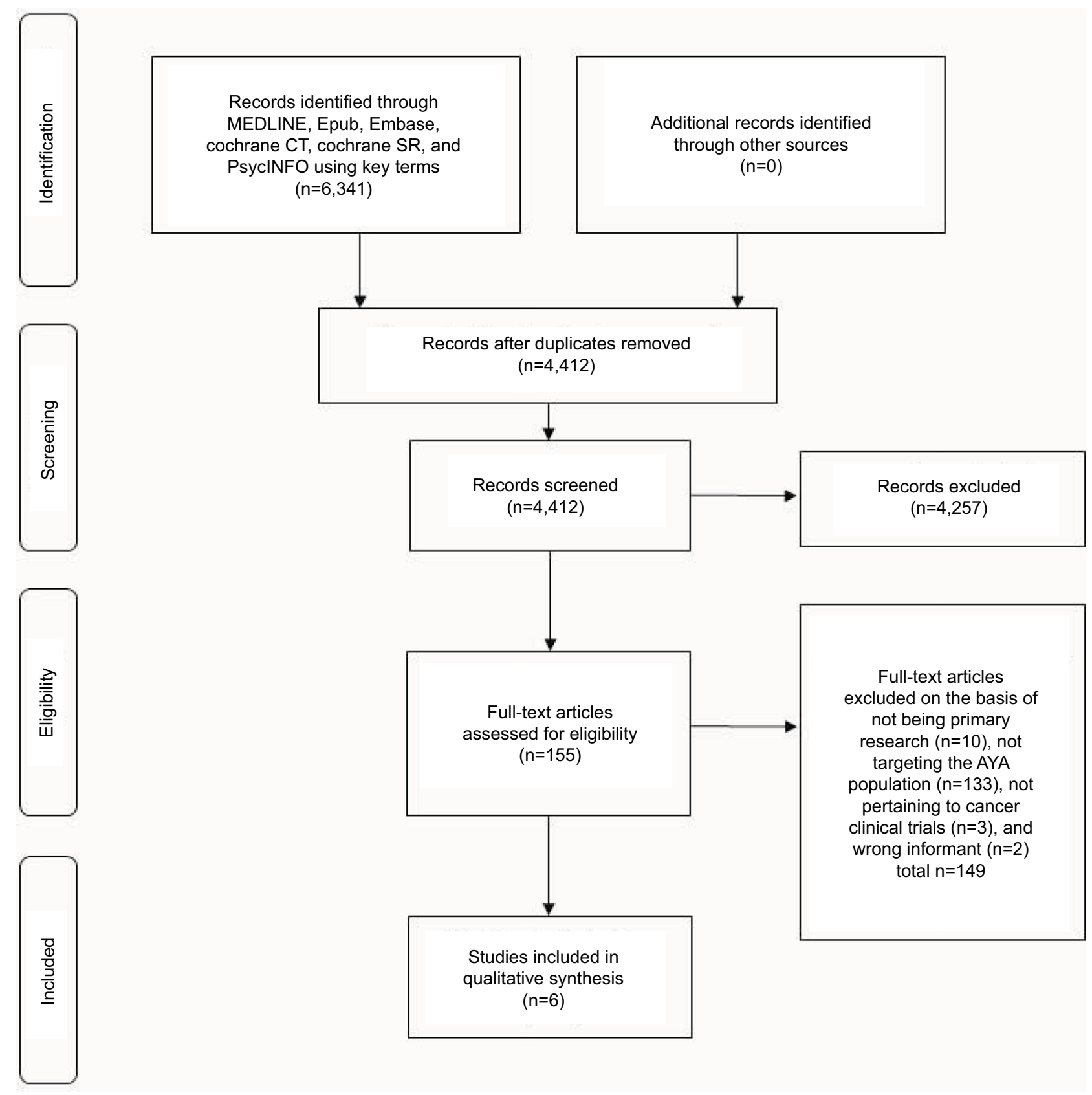

Figure I PRISMA flow diagram of articles identified specifically pertaining to patient-related psychosocial barriers to clinical enrollment in adolescent and young adults with cancer.

Abbreviations: CT, clinical trials; AYA, adolescents and young adults; PRISMA, Preferred Reporting Items for Systematic Reviews and Meta-Analyses.

for CT enrollment was hope for clinical benefit (identified in five of six studies ${ }^{21,22,24-26}$ ), followed by altruism (identified in four of six studies ${ }^{21,24-26}$ ). These studies generally included younger AYA patients ( $\leq 21$ years), and thus also reported involvement of parents and families in the decisionmaking process. Having autonomy was cited in three out of six studies ${ }^{22,25,26}$ as being a positive facilitator to enrollment, with one of those studies being conducted in a pediatric center. ${ }^{25}$ Incentives that were unique to the study conducted in an AYA specialized center were good communication with physician, support of a clinical nurse specialist (CNS), and peer support. ${ }^{26}$

Regarding attitudes to $\mathrm{CT}$ enrollment, major barriers identified were categorized into protocol-, patient-, and physician-related barriers. The most commonly reported themes, cited in four out of six studies, included: fear of potential side effects; $;^{22-24,26}$ prolonged hospitalization; $;^{21,24-26}$ discomfort with experimentation; ${ }^{21-23,26}$ the protocols being too complex; $;^{21,22,25,26}$ and the trial not offering the best option. ${ }^{21-23,25}$ Moreover, physicians were identified as playing 
Table I A summary of the demographics and tools of dedicated research in AYA populations of perceptions and attitudes regarding clinical trial enrollment

\begin{tabular}{|c|c|c|c|c|c|c|c|}
\hline Study & Setting & Aim & $\mathbf{n}$ & Tool useds & Cancer type & Gender & Phase \\
\hline Barakat et al ${ }^{25}$ & $\begin{array}{l}\text { Pediatric } \\
\text { center }\end{array}$ & $\begin{array}{l}\text { To understand decision making of } \\
\text { AYAs, parents, and providers and } \\
\text { evaluate relevance of PRPQ for } \\
\text { measuring attitudes toward Phase } \\
\text { III clinical trial enrollment }\end{array}$ & $\begin{array}{l}13 \\
(15-21- \\
\text { year } \\
\text { olds })\end{array}$ & $\begin{array}{l}\text { Qualitative } \\
\text { interview }\end{array}$ & $\begin{array}{l}\text { 50\% lymphoma/ } \\
\text { leukemia }\end{array}$ & $50 \%$ female & Phase III \\
\hline Grigsby et al ${ }^{22}$ & $\begin{array}{l}\text { Adult center } \\
\text { (patients } \\
\text { recruited from } \\
\text { California } \\
\text { cancer registry) }\end{array}$ & $\begin{array}{l}\text { To assess attitudes of AYAs } \\
\text { compared to healthy college } \\
\text { students toward clinical trial } \\
\text { participation }\end{array}$ & $\begin{array}{l}99 \\
(20-39- \\
\text { year } \\
\text { olds })\end{array}$ & $\begin{array}{l}\text { ACTS-CT } \\
\text { survey }\end{array}$ & $\begin{array}{l}\text { Leukemia/ } \\
\text { lymphoma }\end{array}$ & $53 \%$ female & $N / A$ \\
\hline Miller et a $\left.\right|^{24}$ & $\begin{array}{l}\text { Pediatric } \\
\text { center }\end{array}$ & $\begin{array}{l}\text { To examine AYAs' perspectives } \\
\text { on understanding and decision } \\
\text { making about pediatric Phase I } \\
\text { clinical trials }\end{array}$ & $\begin{array}{l}20 \\
(14-2 \mid- \\
\text { year } \\
\text { olds })\end{array}$ & $\begin{array}{l}\text { Comprehensive } \\
\text { interview }\end{array}$ & $\begin{array}{l}90 \% \text { sarcoma/ } \\
\text { central nervous } \\
\text { system }\end{array}$ & $25 \%$ female & Phase I \\
\hline Pearce et $\mathrm{al}^{26}$ & $\begin{array}{l}\text { AYA specialist } \\
\text { care center }\end{array}$ & $\begin{array}{l}\text { To explore perceptions of AYAs } \\
\text { and professionals in participating } \\
\text { in Phase III bone cancer clinical } \\
\text { trials }\end{array}$ & $\begin{array}{l}21 \\
(15-25- \\
\text { year } \\
\text { olds })\end{array}$ & $\begin{array}{l}\text { Semi-structured } \\
\text { narrative } \\
\text { interview }\end{array}$ & Bone & $33 \%$ female & Phase III \\
\hline Read et $\mathrm{al}^{2 \mathrm{I}}$ & $\begin{array}{l}\text { Pediatric } \\
\text { center }\end{array}$ & $\begin{array}{l}\text { To determine personal factors } \\
\text { that influence AYA decisions to } \\
\text { enroll in clinical trials and whether } \\
\text { these are in line with perceptions } \\
\text { of parents of patients }\end{array}$ & $\begin{array}{l}86 \\
(12-22- \\
\text { year } \\
\text { olds })\end{array}$ & $\begin{array}{l}\text { Self-reported } \\
\text { validated } \\
\text { questionnaire }\end{array}$ & N/A & N/A & $\mathrm{N} / \mathrm{A}$ \\
\hline $\begin{array}{l}\text { Shnorhavorian } \\
\text { et } \mathrm{al}^{23}\end{array}$ & $\begin{array}{l}\text { Adult center } \\
\text { ( } 4 \% \text { pediatric } \\
\text { institute) }\end{array}$ & $\begin{array}{l}\text { To assess AYA awareness of } \\
\text { clinical trial availability and reasons } \\
\text { why AYAs with knowledge of } \\
\text { clinical trials do not participate }\end{array}$ & $\begin{array}{l}515 \\
(15-39- \\
\text { year } \\
\text { olds })\end{array}$ & $\begin{array}{l}\text { Self- } \\
\text { administered } \\
\text { questionnaire }\end{array}$ & $\begin{array}{l}90 \% \text { GCT, } \\
\text { lymphoma }\end{array}$ & $36.5 \%$ female & $\mathrm{N} / \mathrm{A}$ \\
\hline
\end{tabular}

Abbreviations: AYA, adolescents and young adults; CT, clinical trials; PRPQ, pediatric research participation questionnaire; N/A, not available; GCT, germ cell tumor.

a role in AYA decision making to CT enrollment, and feeling coerced by physicians was a reported deterrent in 50\% of studies. ${ }^{21,24,26}$ Other barriers reported in $50 \%$ of studies included: increased discomfort/additional procedures ${ }^{21,25,26}$ and feeling overwhelmed with the amount of information delivered..$^{21,25,26}$

\section{Discussion}

There is a paucity of information on the perceptions that influence accrual among AYA cancer patients, especially in those treated at adult centers. Because existing studies are generally diverse in their inclusion, robust conclusions applicable to wide AYA audiences are impractical. As the majority of AYAs are being cared for within the adult medical oncology system, increasing the understanding of attitudinal factors influencing their participation in trials will identify perceptual gaps that may allow for systematic intervention. AYA are seen in various environments including large tertiary-care cancer centers and smaller community or private oncology practices, within which different strategies for trial accrual are required. In the studies identified in this systematic review, we observed similarities between the AYA population and older adult population. The most cited reasons for accepting enrollment were hope for positive clinical benefit and a feeling of altruism. Of the potential barriers identified among AYA, the most frequently cited items included prolonged hospitalization and being uncomfortable with experimentation.

It is likely that barriers to $\mathrm{CT}$ will differ according to trial stage. Phase I CT are typically complex as they are designed to identify the maximal tolerated doses of investigational agents without clear therapeutic benefit to the individual. There was only one study specific to AYA patients on Phase I trials which reported lack of therapeutic options and side effect profile as strong factors related to $\mathrm{CT}$ acceptability. ${ }^{24}$ Thus, tailoring of consent procedures may be required according to trial phase. Allowing dedicated communication strategies for AYA in these trials, ${ }^{20}$ having access to multidisciplinary teams ${ }^{27}$ or using multimedia strategies for clinicians ${ }^{28}$ may help AYA in supporting their $\mathrm{CT}$ decisions (Figure 2). In contrast, Phase III studies typically are aimed to 
Table 2 A summary of dedicated research in AYA populations of perceptions and attitudes regarding clinical trial enrollment

\begin{tabular}{|c|c|c|c|c|c|c|}
\hline \multirow{2}{*}{$\begin{array}{l}\text { Location } \\
\text { Study }\end{array}$} & \multicolumn{3}{|c|}{ Pediatric center } & \multirow{2}{*}{$\begin{array}{l}\text { AYA center } \\
\text { Pearce } \\
\text { et } \mathrm{al}^{26}\end{array}$} & \multicolumn{2}{|c|}{ Adult center } \\
\hline & $\begin{array}{l}\text { Barakat } \\
\text { et } \mathrm{al}^{25}\end{array}$ & $\begin{array}{l}\text { Miller } \\
\text { et } \mathrm{al}^{24}\end{array}$ & $\begin{array}{l}\text { Read } \\
\text { et } \mathbf{a l}^{21}\end{array}$ & & $\begin{array}{l}\text { Grigsby } \\
\text { et } \mathbf{a l}^{22}\end{array}$ & $\begin{array}{l}\text { Shnorhavorian } \\
\text { et } \mathrm{al}^{23}\end{array}$ \\
\hline \multicolumn{7}{|l|}{ Perceived barriers } \\
\hline \multicolumn{7}{|l|}{ Protocol-related barriers } \\
\hline Dislike idea of randomization & & & & • & & - \\
\hline Protocol too complex & - & & - & - & - & \\
\hline Worry about side effects & & • & & • & • & • \\
\hline Trial/treatment does not offer best option & • & & • & & • & $\bullet$ \\
\hline Treatment insufficiently tested & & & • & & • & • \\
\hline Too much time/prolonged hospitalization & • & • & • & • & & \\
\hline Increased discomfort/additional procedures & - & & - & • & & \\
\hline Decision to enroll at time of diagnosis & - & & & • & & \\
\hline Fear-provoking terminology & & & & • & & \\
\hline \multicolumn{7}{|l|}{ Patient-related barriers } \\
\hline Uncomfortable with experimentation & & & • & • & • & • \\
\hline Quality of life might be reduced & • & & & & & \\
\hline Lack of family/peer support & & & & • & & \\
\hline Overwhelmed with amount of information & • & & • & • & & \\
\hline \multicolumn{7}{|l|}{ Physician-related barriers } \\
\hline Feeling coerced to join & & • & • & • & & \\
\hline Physicians' attitude toward trial & & & & • & - & \\
\hline Need to switch physicians & & & & & & • \\
\hline \multicolumn{7}{|l|}{ Perceived facilitators } \\
\hline Positive clinical affect & • & • & • & • & - & \\
\hline Need for an option & & • & & & & \\
\hline Altruism & - & • & • & • & & \\
\hline Best treatment option & & • & & & • & \\
\hline Improve quality of life & & • & & & & \\
\hline Safety/closely monitored & & & & & • & \\
\hline Having autonomy & • & & & • & • & \\
\hline Support of peers & & & & • & - & \\
\hline Good communication with physician & & & & • & & \\
\hline Support of clinical nurse specialist & & & & • & & \\
\hline
\end{tabular}
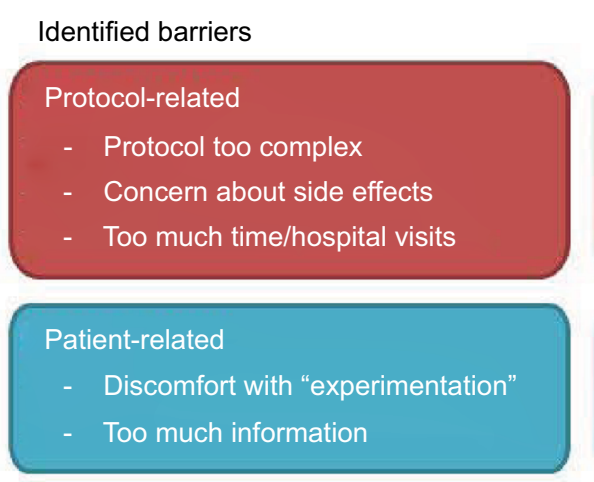

\section{Physician-related}

Feelings of coercion

Physicians' attitudes toward trial

\section{Possible solutions}

Use of multimedia strategies to help explain protocols

Tailoring of consent for AYA based on trial phase

Access to neutral multidisciplinary teams when possible to facilitate further trial discussions

Advocate for trial design to have increased flexibility of visits

Use of multimedia strategies to explain trials

Access to neutral multidisciplinary teams

Involving family in decision making

- Improve physician education on the importance of patientphysician relationship with respect to AYA

- Access to neutral or AYA multidisciplinary teams

- Multimedia strategies for clinicians

Figure 2 Selected examples of barriers to clinical trials in AYA highlighting some potential solutions.

Abbreviation: AYA, adolescents and young adults. 
show survival improvements of an experimental agent compared to standard of care. Thus, CT acceptability will likely be related to the underlying efficacy of the control arm. In the two Phase III studies, ${ }^{25,26}$ the perceived benefit outweighed the burden, but there were challenges at determining ideal degree of parental involvement in the decision making, and both studies reported having individual autonomy as an incentive to enrollment. In both studies, the upper age limit was 25 years; thus, the extent to which family involvement is wanted may vary in older AYA.

The role of the parent or family is crucial when considering the developmental stage of AYA. ${ }^{29-32}$ As the adolescent developmental period is categorized by significant physical, emotional, pubertal, and cognitive changes, a thorough developmental assessment is important as they may have significant bearing on CT acceptability. In addition, a cancer diagnosis in this age group typically coincides with the development of self-determination. Where exactly individuals fall within the developmental stage spectrum (e.g., autonomy versus dependence) may directly influence accrual to $\mathrm{CT} .{ }^{9}$ This presents a challenge in determining how much of a role AYA have, or should have, in the CT enrollment decision-making process. Of note, Pearce et al reported that AYA emphasized the importance of having autonomy in decision-making. ${ }^{26}$ Moreover, AYA patients in the study by Barakat et al reported that their involvement in decisionmaking with regard to CT was limited, and they expressed a desire to not feel dependent upon their parents. ${ }^{25}$ An association between involvement in the decision to enroll in CT and participating in those trials was identified; however, the extent of this association is uncertain. ${ }^{25}$ Conversely, in the study by Miller et al, $85 \%$ of adolescents reported being the final decision-maker in regard to CT enrollment. ${ }^{24}$ Beliefs with regard to the level of AYA involvement in decision making from the perspective of health care professionals or parents were mixed. ${ }^{24,25}$ A concern cited was that health care professionals might be underestimating the role of adolescents in the decision-making process, resulting in less effective communication about trials (i.e., information targeted more toward parents than patients). ${ }^{33}$ Half of the studies cited patient autonomy as a facilitator of CT enrollment for AYA..$^{22,25,26}$ The findings of these studies address the importance of further investigation on autonomy in decision making in this unique age group.

In contrast to children, AYA may experience higher rates of general distress due to a heightened understanding of the severity of their diagnosis as well as increased anxiety concerning lifestyle changes and premature mortality. ${ }^{32,34,35}$
Although a cancer diagnosis in the AYA years occurs during a time of vulnerability and, thus, may be associated with psychological maladjustment, depression, anxiety, cancer denial, or distress, there is no information as to how this may interact with $\mathrm{CT}$ comprehension and acceptability. ${ }^{9}$ In addition, younger adolescents ( $<18$ years) have shown poor understanding of $\mathrm{CT}$, and, as a result, a reduced willingness to participate. ${ }^{9,36}$ Being in a stage of developmental transition, AYAs may not have fully developed executive functioning skills, such as planning and impulse control, which are critical in making the decision to enroll., ${ }^{9,37}$ This again illuminates the need for further investigation into the appropriate degree of involvement in CT decision-making of AYA of different ages.

In contrast, CT acceptability in older adults has been well researched. Commonly cited reasons for participation include increased hope and altruism. ${ }^{4}$ Altruism and positive clinical effect were the most cited in the AYA population. As for barriers towards enrollment identified in adult populations, these include concerns regarding research process/ protocol, ${ }^{17}$ potential side effects, ${ }^{17}$ fear of the unknown, ${ }^{38}$ lack of control, ${ }^{38}$ quality of life/autonomy loss, ${ }^{9}$ belief that risks outweighed benefits, ${ }^{39}$ and that the $\mathrm{CT}$ researcher was more interested in research than the patient. ${ }^{9,39}$ This granular level of understanding of trial perceptions and attitudes is lacking in the AYA literature, and a focus of how AYA perceptions change as individuals age is being investigated by our group..$^{40}$ In the studies analyzed, enrollment barriers common to both AYA and older adult populations included worries about potential side effects, concerns regarding protocol, insufficient testing, and quality of life. Barriers found to be unique to, or more prevalent among, AYA included the prolonged time commitment required in $\mathrm{CT}$, which was not as significant for older adults, ${ }^{17}$ and a lack of peer support. ${ }^{26} \mathrm{~A}$ participant in the study by Pearce et al reflected that a sense of lost time is more acute for a younger patient than it is for an older adult. ${ }^{26}$ Where possible, future trial design may need to consider increased flexibility of clinical visits in order to address this barrier (Figure 2).

AYA are a unique patient population and, therefore, require specialized care. Of the six identified studies dedicated to the AYA population, half were conducted in pediatric institutions. All of the participants in the only study conducted in an AYA center emphasized the importance of being treated in a specialized care setting. ${ }^{26}$ Interacting with patients around their own age and having health care personnel trained in communicating with them were considered imperative. Furthermore, this study found that patients greatly valued the CNS in helping to make their decision to participate in CTs and were viewed 
as a neutral party who is pivotal in providing or clarifying information and offering support. ${ }^{26}$ This finding speaks to the importance of AYA having care provided by a specialized team trained in dealing with their specific age group.

Communication with health care providers and the strength of the relationship between patient and provider is unique for AYA when compared to children or older adults. Doctor-patient relationships may be particularly important for AYA and may assist with treatment-related decisions, delivery of developmentally and culturally appropriate information, and management of preference for information delivery. Medical provider knowledge of CT availability and communication may affect AYA awareness level, ${ }^{10}$ with three of the studies assessing whether AYAs were aware of CTs being available. For example, Shnorhavorian et al found that only $17 \%$ reported knowledge of a $\mathrm{CT}^{23}$ Once health care professionals are well informed on CT availability, communicating this information to patients effectively is an additional challenge that requires on understanding of the unique needs and perceptions of AYA. In the studies analyzed, being overwhelmed by too much information was cited as a barrier to enrollment. Terminology was another perceived barrier for AYA (i.e., using the word "trial" instead of "study"). These findings can be used to tailor the informed consent process to be more appealing to AYA.

It is evident that an enhanced understanding of psychosocial barriers to CT enrollment of AYA is needed in order to help guide future interventions and increase accrual in this population. The six studies identified in the systematic review are limited by small sample size, bias toward specific cancers, and half of the studies being conducted in pediatric centers. Future studies of AYAs in larger adult-based cohorts with extended age ranges should be conducted in order to assess AYA involvement in decision-making for CT enrollment, identify differences across the AYA spectrum, isolate means to incentivize $\mathrm{CT}$ enrollment, and assess the impact of AYA-specialized care. Increasing CT enrollment in this population can ultimately lead to better understanding and care of this complex patient group.

\section{Conclusion}

Limited information is available with regard to the perceptions of and attitudes toward CT acceptability among AYA patients. In addition to addressing regulatory and infrastructural barriers, attitudes toward trials are an important consideration for the AYA oncology community. Future large-cohort studies should be addressed toward understanding of this and to describe the role of dedicated AYA programs to facilitate these discussions and spearhead multidisciplinary conversations, specifically for CT accrual. Moreover, an increased role for peer support networks (leveraging existing frameworks) and the development of novel communication strategies are deserving of further exploration. More studies among AYA treated within adult oncology cancer settings are required, and studies using mixed methods approaches with a more diverse demographic may be informative. With a more comprehensive understanding of perceptions and attitudes surrounding trials, systematic interventions can be designed.

\section{Acknowledgments}

This paper was supported by the Michael Kamin Hart Fund at Princess Margaret Cancer Center. Given that this is a systematic review of published literature, the authors do not control the primary data of these original papers. The abstract of this paper was presented at the 2nd Global AYA Cancer Congress, December 5-7, 2017, Atlanta, USA, as a poster presentation with interim findings.

\section{Disclosure}

Abha A Gupta and Jeremy Lewin share senior authorship. The authors report no conflicts of interest in this work.

\section{References}

1. Warner EL, Kent EE, Trevino KM, Parsons HM, Zebrack BJ, Kirchhoff AC. Social well-being among adolescents and young adults with cancer: a systematic review. Cancer. 2016;122(7):1029-1037.

2. Fern LA, Whelan JS. Recruitment of adolescents and young adults to cancer clinical trials - international comparisons, barriers, and implications. Semin Oncol. 2010;37(2):e1-e8.

3. Downs-Canner S, Shaw PH. A comparison of clinical trial enrollment between adolescent and young adult (AYA) oncology patients treated at affiliated adult and pediatric oncology centers. J Pediatr Hematol Oncol. 2009;31(12):927-929.

4. Jenkins V, Farewell V, Farewell D, et al; TTT Steering committee. Drivers and barriers to patient participation in RCTs. $\mathrm{Br} J$ Cancer. 2013;108(7):1402-1407.

5. Peppercorn JM, Weeks JC, Cook EF, Joffe S. Comparison of outcomes in cancer patients treated within and outside clinical trials: conceptual framework and structured review. Lancet. 2004;363(9405):263-270.

6. Edwards SJ, Braunholtz DA, Lilford RJ, Stevens AJ. Ethical issues in the design and conduct of cluster randomised controlled trials. BMJ. 1999;318(7195):1407-1409.

7. Unger JM, Barlow WE, Martin DP, et al. Comparison of survival outcomes among cancer patients treated in and out of clinical trials. $J$ Natl Cancer Inst. 2004;106(3):dju002.

8. Smith AW, Seibel NL, Lewis DR, et al. Next steps for adolescent and young adult oncology workshop: an update on progress and recommendations for the future. Cancer. 2016;122(7):988-999.

9. Buchanan ND, Block R, Smith AW, Tai E. Psychosocial barriers and facilitators to clinical trial enrollment and adherence for adolescents with cancer. Pediatrics. 2014;133 Suppl 3:S123-S130.

10. Parsons HM, Harlan LC, Seibel NL, Stevens JL, Keegan TH. Clinical trial participation and time to treatment among adolescents and young adults with cancer: does age at diagnosis or insurance make a difference? J Clin Oncol. 2011;29(30):4045-4053. 
11. Burke ME, Albritton K, Marina N. Challenges in the recruitment of adolescents and young adults to cancer clinical trials. Cancer. 2007;110(11): 2385-2393.

12. Klamerus JF, Bruinooge SS, Ye X, et al. The impact of insurance on access to cancer clinical trials at a comprehensive cancer center. Clin Cancer Res. 2010;16(24):5997-6003.

13. Cassileth BR, Lusk EJ, Miller DS, Hurwitz S. Attitudes toward clinical trials among patients and the public. JAMA. 1982;248(8):968-970.

14. Fallowfield LJ, Jenkins V, Brennan C, Sawtell M, Moynihan C, Souhami RL. Attitudes of patients to randomised clinical trials of cancer therapy. Eur J Cancer. 1998;34(10):1554-1559.

15. Ellis PM, Dowsett SM, Butow PN, Tattersall MH. Attitudes to randomized clinical trials amongst out-patients attending a medical oncology clinic. Health Expect. 1999;2(1):33-43.

16. Townsley CA, Selby R, Siu LL. Systematic review of barriers to the recruitment of older patients with cancer onto clinical trials. $J$ Clin Oncol. 2005;23(13):3112-3124.

17. Mills EJ, Seely D, Rachlis B, et al. Barriers to participation in clinical trials of cancer: a meta-analysis and systematic review of patient-reported factors. Lancet Oncol. 2006;7(2):141-148.

18. Siembida EJ, Bellizzi KM. The doctor-patient relationship in the adolescent cancer setting: a developmentally focused literature review. J Adolesc Young Adult Oncol. 2015;4(3):108-117.

19. Tong A, Sainsbury P, Craig J. Consolidated criteria for reporting qualitative research (COREQ): a 32-item checklist for interviews and focus groups. Int J Qual Health Care. 2007;19(6):349-357.

20. Baker JN, Leek AC, Salas HS, et al. Suggestions from adolescents, young adults, and parents for improving informed consent in phase 1 pediatric oncology trials. Cancer. 2013;119(23):4154-4161.

21. Read K, Fernandez CV, Gao J, et al. Decision-making by adolescents and parents of children with cancer regarding health research participation. Pediatrics. 2009;124(3):959-965.

22. Grigsby TJ, Kent EE, Montoya MJ, et al. Attitudes toward cancer clinical trial participation in young adults with a history of cancer and a healthy college student sample: a preliminary investigation. J Adolesc Young Adult Oncol. 2014;3(1):20-27.

23. Shnorhavorian M, Doody DR, Chen VW, et al; AYA HOPE Study Collaborative Group. Knowledge of clinical trial availability and reasons for nonparticipation among adolescent and young adult cancer patients: a population-based study. Am J Clin Oncol. Epub 2016 Sep 15.

24. Miller VA, Baker JN, Leek AC, et al. Adolescent perspectives on phase I cancer research. Pediatr Blood Cancer. 2013;60(5):873-878.

25. Barakat LP, Schwartz LA, Reilly A, Deatrick JA, Balis F. A qualitative study of phase III cancer clinical trial enrollment decision-making: perspectives from adolescents, young adults, caregivers, and providers. J Adolesc Young Adult Oncol. 2014;3(1):3-11.
26. Pearce S, Brownsdon A, Fern L, Gibson F, Whelan J, Lavender V. The perceptions of teenagers, young adults and professionals in the participation of bone cancer clinical trials. Eur J Cancer Care (Engl). Epub 2016 Mar 3.

27. Tan GH, Totapally BR, Torbati D, Wolfsdorf J. End-of-life decisions and palliative care in a children's hospital. $J$ Palliat Med. 2006;9(2): 332-342.

28. Yap TY, Yamokoski A, Noll R, Drotar D, Zyzanski S, Kodish ED. A physician-directed intervention: teaching and measuring better informed consent. Acad Med. 2009;84:1036-1042.

29. Portteus A, Ahmad N, Tobey D, Leavey P. The prevalence and use of antidepressant medication in pediatric cancer patients. $J$ Child Adolesc Psychopharmacol. 2006;16(4):467-473.

30. Seitz DC, Besier T, Goldbeck L. Psychosocial interventions for adolescent cancer patients: a systematic review of the literature. Psychooncology. 2009;18(7):683-690.

31. Soliman H, Agresta SV. Current issues in adolescent and young adult cancer survivorship. Cancer control. 2008;15(1):55-62.

32. Zebrack BJ, Chesler MA, Penn A. Psychosocial support. In: Bleyer WA, Barr RD, editors. Cancer in Adolescents and Young Adults. New York: Springer Verlag; 2007:375-385.

33. de Vries MC, Wit JM, Engberts DP, Kaspers GJ, van Leeuwen E. Pediatric oncologists' attitudes towards involving adolescents in decisionmaking concerning research participation. Pediatr Blood Cancer. 2010;55(1):123-128.

34. Evan EE, Kaufman M, Cook AB, Zeltzer LK. Sexual health and selfesteem in adolescents and young adults with cancer. Cancer. 2006;107(7 Suppl):1672-1679.

35. Zeltzer LK. Cancer in adolescents and young adults psychosocial aspects. Long-term survivors. Cancer. 1993;71(10 Suppl):3463-3468.

36. Lara PN Jr, Paterniti DA, Chiechi C, et al. Evaluation of factors affecting awareness of and willingness to participate in cancer clinical trials. J Clin Oncol. 2005;23(36):9282-9289.

37. Giedd JN. The teen brain: insights from neuroimaging. JAdolesc Health. 2008;42(4):335-343.

38. Quinn GP, Koskan A, Wells KJ, et al. Cancer patients' fears related to clinical trial participation: a qualitative study. J Cancer Educ. 2012;27(2): 257-262.

39. Crosson K, Eisner E, Brown C, Ter Maat J. Primary care physicians' attitudes, knowledge, and practices related to cancer clinical trials. $J$ Cancer Educ. 2001;16(4):188-192.

40. Gupta A, Bell JA, Wang K, et al. Evaluation of adolescents and young adults (AYA) attitudes towards participation in cancer clinical trials. Presented at: the American Society of Clinical Oncology Annual Meeting 2017. J Clin Oncol. 2017;35:10047.
Adolescent Health, Medicine and Therapeutics

Publish your work in this journal

Adolescent Health, Medicine and Therapeutics is an international, peer-reviewed, open access journal focusing on health, pathology, and treatment issues specific to the adolescent age group. All aspects of health maintenance, preventative measures and disease treatment interventions are addressed within the journal and practitioners from all disciplines are
Dovepress

invited to submit their work as well as healthcare researchers and patient support groups. This journal is included in PubMed. The manuscript management system is completely online and includes a very quick and fair peer-review system. Visit http://www.dovepress.com/testimonials. php to read real quotes from published authors. 